\title{
Cardiac Dedicated Ultrafast SPECT Cameras: New Designs and Clinical Implications
}

\author{
Ernest V. Garcia, Tracy L. Faber, and Fabio P. Esteves \\ Department of Radiology, Emory University School of Medicine, Atlanta, Georgia
}

\begin{abstract}
Learning Objectives: On successful completion of this activity, participants should be able to describe (1) the differences in imaging performance between new solid-state ultrafast cardiocentric imaging devices and conventional dual-detector rotating SPECT cameras, and (2) the clinical trials that confirm the diagnostic accuracy of the new ultrafast cardiocentric imaging devices.

Financial Disclosure: The authors of this article have indicated that they received a research grant from GE Healthcare to evaluate the Discovery NM $530 \mathrm{c}$ SPECT system. No other relevant relationships that could be perceived as a real or apparent conflict of interest were reported.

CME Credit: SNM is accredited by the Accreditation Council for Continuing Medical Education (ACCME) to sponsor continuing education for physicians. SNM designates each JNM continuing education article for a maximum of 1.0 AMA PRA Category 1 Credit. Physicians should claim only credit commensurate with the extent of their participation in the activity.

For CE credit, participants can access this activity through the SNM Web site (http://www.snm.org/ce_online) through February 2012.
\end{abstract}

Myocardial perfusion imaging (MPI) using nuclear cardiology techniques has been widely applied in clinical practice because of its well-documented value in the diagnosis and prognosis of coronary artery disease. Industry has developed innovative designs for dedicated cardiac SPECT cameras that constrain the entire detector area to imaging just the heart. New software that recovers image resolution and limits image noise has also been implemented. These SPECT innovations are resulting in shortened study times or reduced radiation doses to patients, promoting easier scheduling, higher patient satisfaction, and, importantly, higher image quality. This article describes these cardiocentric SPECT software and hardware innovations, which provide a strong foundation for the continued success of myocardial perfusion SPECT.

Key Words: cardiac dedicated ultrafast SPECT cameras; camera; cardiac

J Nucl Med 2011; 52:210-217

DOI: 10.2967/jnumed.110.081323

\section{$\mathbf{M}$}

yocardial perfusion SPECT is often used in the noninvasive assessment of hemodynamically significant coronary artery disease. Despite its success, current clinical, scientific, and financial needs require that hardware and software be further improved to enable myocardial perfusion SPECT to answer these challenges of modern health care. It is difficult to realize these improvements with the imaging hardware and software used in most nuclear cardiology laboratories today. The basic SPECT camera de-

Received Oct. 14, 2010; revision accepted Dec. 1, 2010.

For correspondence contact: Ernest V. Garcia, Department of Radiology, Emory University Hospital, 1364 Clifton Rd., Atlanta, GA 33022.

E-mail: ernest.garcia@emory.edu

COPYRIGHT @ 2011 by the Society of Nuclear Medicine, Inc. sign with standard parallel-hole collimators is more than $50 \mathrm{y}$ old (1) and is inefficient because only a small portion of the available $\mathrm{NaI}$ crystal detector area is used to image the heart (Fig. 1A). The basic filtered backprojection reconstruction algorithm used by most SPECT systems today is even older, dating to more than $90 \mathrm{y}$ ago (2). This article describes the design of new heart-dedicated ultrafast SPECT cameras for MPI and discusses how these devices, together with new reconstruction algorithms, are poised to meet the present health-care challenges by improving image quality while reducing study time, radiation dose to the patient, and, ultimately, study cost.

\section{ADVANCES IN IMAGE RECONSTRUCTION}

Recent software improvements in iterative image reconstruction take into account the loss of resolution with distance inherent in parallel-hole collimators. Using this knowledge in conjunction with the imaging properties of the system allows for a mathematic correction of this resolution degradation known as resolution recovery. At the same time, noise is suppressed because additional counts are now correctly accounted for instead of being treated as noise. Moreover, noise regularization techniques have been implemented that go beyond simple smoothing by considering the expected noise for the regional count density $(3,4)$. Because resolution recovery actually reduces noise while improving spatial resolution, as compared with filtered backprojection, resolution recovery can yield reconstructed images from studies acquired in less time with the same signal-to-noise ratio as filtered backprojection images reconstructed from studies acquired for longer times.

Because resolution recovery requires specific information as to the imaging properties of the system, the recovery algorithm must accurately account for the physical characteristics of the detector, the collimator, and the patient. 

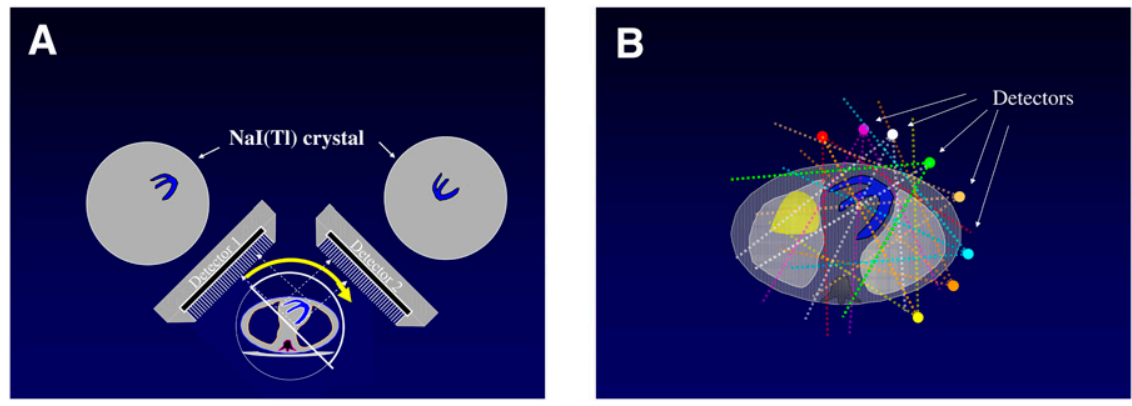

FIGURE 1. (A) Conventional camera design in dual-detector SPECT systems is more than 50 y old and, when applying standard parallel-hole collimators, is limited to imaging heart using only small portion of available useful area of $\mathrm{Nal}(\mathrm{TI})$ crystal detectors. (B) In new-generation dedicated cardiac ultrafast-acquisition scanners, 8 detectors surrounding patient simultaneously image heart. These new designs vary in number and type of scanning or stationary detectors and in whether Nal or CZT solid-state detectors are used. They all have in common the potential for 5 - to 10-fold increase in count sensitivity at no loss of, or even at gain in, resolution.

These recovery algorithms use a database of known detector and collimator characteristics. Recovery also requires a specific description of the orbit shape, radius, or distance from the patient to the detector.

Most manufacturers of SPECT cameras have implemented some version of resolution recovery or noise reduction algorithms into software for maximum-likelihood expectation maximization or ordered-subset expectation maximization iterative reconstruction, keeping the exact details proprietary. The algorithms that have been developed for conventional SPECT cameras include Astonish (Philips) (5), Flash3D (Siemens Medical Solutions) (6), Evolution (GE Healthcare) (3), nSPEED (Digirad Corp.) (7), and wide-beam reconstruction by a third-party vendor (UltraSPECT) $(3,4,8)$.

Each of these algorithms has been clinically validated to various degrees. The clinical trials compared conventional SPECT reconstruction with the resolution recovery/noise reduction reconstruction of MPI studies and showed that the latter...

- may be acquired in half the time without compromising qualitative or quantitative diagnostic performance (3-5), with or without attenuation correction (5);

- may even be acquired in a quarter of the time if the reconstruction is optimized for the reduced count density (8);

- may be acquired in half the time while improving functional diagnostic performance (4);

- provide left ventricular volumes that correlate but are significantly smaller (4);

- provide left ventricular ejection fractions that correlate but are lower than conventional reconstruction because of a reduction in end-diastolic volume (4) or, for some implementations, an increase in end systolic volume (4); and

- provide similar diagnostic quality whether imaging time is reduced by half or a half-dose is injected (4).

Importantly, variations of these resolution recovery and noise reduction algorithms have been implemented in the new ultrafast camera designs to further improve image spatial and contrast resolution while reducing count noise.
Figure 2 compares patient images reconstructed with standard ordered-subset expectation maximization to the halftime acquisition with wide-beam reconstruction.

\section{NEW ULTRAFAST CAMERA DESIGNS}

Several manufacturers have begun to break away from the conventional SPECT approach to create innovatively designed dedicated cardiac cameras. These camera designs have in common that all available detectors are constrained to imaging just the cardiac field of view. Figure 1B shows how 8 detectors surrounding the patient are all simultaneously imaging the heart. These new designs vary in the number and type of scanning or stationary detectors and in whether NaI, CsI, or cadmium-zinc-telluride (CZT) solid-state detectors are used. They all have in common the potential for a 5- to 10-fold increase in count sensitivity at no loss of, or even a gain in, resolution, resulting in the potential for acquiring a stress myocardial perfusion scan in $2 \mathrm{~min}$ or less if the patient is injected with a standard dose. Some of this gain in sensitivity can be traded for a linear reduction in the injected dose to reduce the patient's exposure to radiation. Thus, in an ultrafast camera with a 10 -fold increase in sensitivity using conventional radiopharmaceutical doses, the dose could be reduced by half and a 5 -fold increase in sensitivity still be maintained.

\section{CZT Solid-State, Multiple Scanning Detector Design (D-SPECT)}

The first SPECT system to offer a totally different design was D-SPECT, manufactured by Spectrum Dynamics (9-12). This system uses solid-state detectors in the form of CZT mounted on 9 vertical columns and placed using $90^{\circ}$ geometry. Each of the 9 detector assemblies is equipped with a square tungsten parallel-hole collimator. Each square hole in the collimator is $2.46 \mathrm{~mm}$ on its side-large in comparison to conventional collimators-and contributing to the increased count sensitivity of the camera. Each detector assembly fans in synchrony with the other 8 detector assemblies while all 9 are simultaneously imaging the heart. The patient is imaged sitting in a reclining position, similar to a dentist's chair, with the patient's left arm placed on top of the detector housing.

Data are acquired by first obtaining a 1-min scout scan for the 9 detectors to identify the location of the heart and 
FIGURE 2. Comparison of standard iterative reconstruction versus resolution recovery applied to half-time acquisitions in healthy patient: stress (top) and rest (bottom) horizontal long-axis slices reconstructed with ordered-subset expectation maximization (A); end-diastolic (top) and end-systolic (bottom) vertical and horizontal long-axis slices reconstructed with ordered-subset expectation maximization (B); stress and rest HLA slices from same patient, acquired for half time, and reconstructed with wide-beam reconstruction (C); and half-time end-diastolic and end-systolic frames reconstructed with wide-beam reconstruction (D). Perfusion distributions and functional information are similar in the 2 reconstructions, whereas half-time wide-beam reconstruction images have higher resolution and higher left ventricular chamber contrast. (Images courtesy of E. Gordon
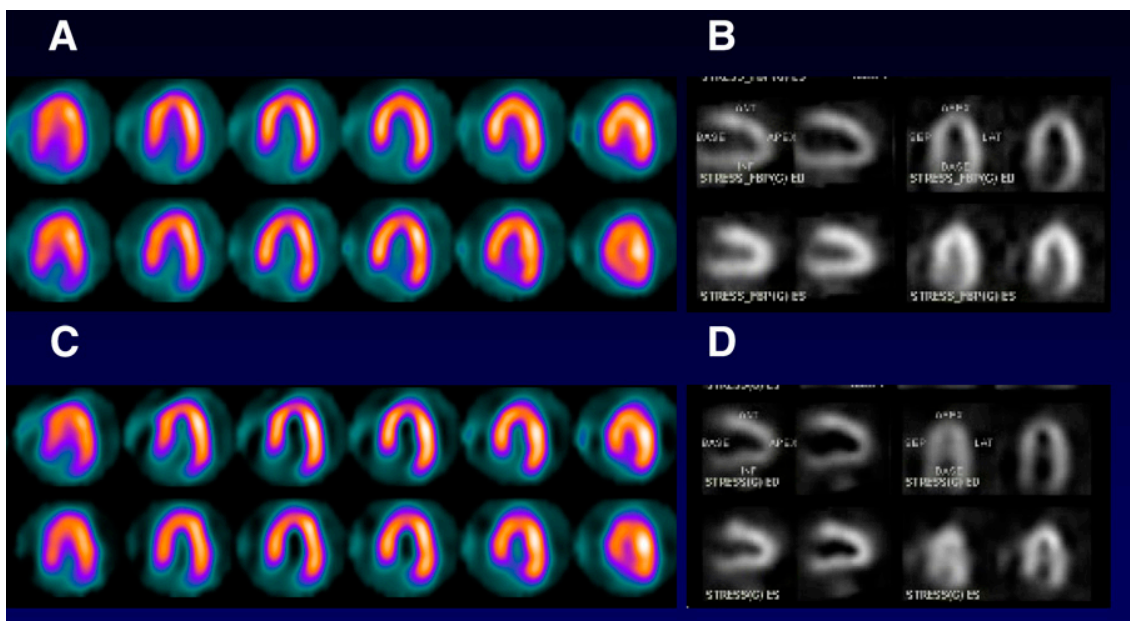

Depuey.)

set the limits of the detectors' fanning motion. The diagnostic scan is then performed with each detector assembly fanning within the limits determined by the scout scan. Reconstruction is performed using a modified iterative algorithm that compensates for the loss of spatial resolution that results from using large, square holes in the collimator by mathematically modeling the acquisition and collimator geometry.

\section{D-SPECT Validation}

Initially, in a single-center clinical trial publication, it was concluded that using a stress-rest ${ }^{99 m}$ Tc MPI protocol and 4- and 2-min D-SPECT acquisitions yielded studies that correlated strongly with 16- and 12-min, respectively, stress-rest conventional SPECT and had equivalent levels of diagnostic performance (9). A subsequent multicenter trial using D-SPECT showed that the use of a device-specific reference database quantitative analysis and a comparison protocol similar to the previous report correlated well with quantitative analysis of conventional SPECT MPI (10). Another study showed the feasibility of performing a fast sequential dual-isotope stress ${ }^{201} \mathrm{Tl} /$ rest ${ }^{99 \mathrm{~m}} \mathrm{Tc}$ protocol with D-SPECT, which could be accomplished in 20 min with image quality and dose similar to those of conventional rest-stress ${ }^{99 \mathrm{~m} T c}$ studies (11). Another small, single-center trial showed that the higher energy resolution of the CZT detectors could be used with the D-SPECT device to perform simultaneous ${ }^{201} \mathrm{Tl}$ (rest)/ 99mTc-sestamibi (stress) 15-min acquisitions that compared favorably in both diagnostic accuracy and image quality with sequential acquisitions obtained with conventional SPECT (12).

\section{CZT Solid-State Multiple-Pinhole Detector Design (Discovery NM 530c)}

The second SPECT system to offer a revolutionary design was the system developed by GE Healthcare (13-
20) known as the Discovery NM 530c. The SPECT design uses Alcyone technology, consisting of an array of 19 pinhole collimators, each with 4 solid-state CZT pixilated detectors, with all 19 pinholes simultaneously imaging the heart with no moving parts during data acquisition. Nine of the pinhole detectors are oriented perpendicular to the patient's long axis whereas 5 are angulated above and 5 below the axis for a true 3-dimensional acquisition geometry. The use of simultaneously acquired views improves the overall sensitivity and gives the complete and consistent angular data needed both for dynamic studies and for the reduction of motion artifacts. In addition, attenuation artifacts may be reduced because not all views are through the attenuator-some may view the heart from above or below. The detector assembly is mounted on a gantry that allows for patient positioning in the supine or prone position. A hybrid system (570c) is also available with a volumetric CT scanner to facilitate attenuation correction and $\mathrm{CT}$ applications. Iterative reconstruction adapted to this geometry is used to create transaxial slices of the heart and to perform attenuation correction.

The use of CZT detectors improves the energy, spatial resolution, and contrast resolution of these imaging systems through direct conversion of the energy and location of the detected photon into an electronic pulse, in contrast to the indirect conversion used in conventional $\mathrm{NaI}(\mathrm{Tl})$ detector cameras. In NaI cameras, the energy of the $\gamma$-ray is first absorbed by the crystal and converted to a large number of visible photons that have to leave the crystal to be detected by all photomultiplier tubes, which then convert the photons to individual electronic pulses. The sum of the pulses from all photomultiplier tubes is used as an energy signal, and the weighted sum is used as the position of the event in the crystal. Any loss of visible photons contributes to miscalculation of the energy and location of the event. In the CZT direct conversion scheme, the $\gamma$-ray is ideally 
absorbed by 1 of the 2.46-mm pixels and directly converted to an electric pulse providing both the energy and the location of the event (Fig. 3). Interestingly, both this Alcyone system and the D-SPECT system use the same CZT detectors from the same manufacturer, with the Discovery system using $76(19 \times 4)$ detectors and the D-SPECT using 36 $(9 \times 4)$. Compared with a state-of-the-art conventional SPECT system, the Alcyone system has been shown to have 1.65 times better energy resolution (5.7\% vs. $9.4 \%$ ), approximately twice better spatial resolution ( $5 \mathrm{~mm}$ vs. 11 $\mathrm{mm}$ central), and 5 (cardiac phantom) to 10 (point source) times more count sensitivity (13).

\section{Discovery NM 530c Validation}

The first multicenter trial demonstrated that using a conventional 1-d ${ }^{99 \mathrm{~m}}$ Tc-tetrofosmin rest-stress MPI protocol and 4- and 2-min Alcyone acquisitions yielded studies that diagnostically agreed $90 \%$ of the time with 14- and 12min, respectively, rest-stress conventional SPECT acquisitions (14). Figure 4, of a hypoperfused patient, compares Discovery NM 530c images with conventional SPECT images acquired with this protocol. Importantly, this trial also showed excellent left ventricular ejection fraction correlations between the Discovery NM 530c and conventional SPECT for rest $(r=0.93, P<0.001)$ and stress $(r=0.91$, $P<0.001)$ gated MPI studies (14). A subsequent singlecenter trial was performed using a $1-\mathrm{d} 99 \mathrm{~m}$ Tc-tetrofosmin adenosine-stress-rest MPI protocol and a 3-min scan for stress and $2 \mathrm{~min}$ for rest using the Discovery NM 530c compared with 15-min conventional SPECT acquisitions for stress and rest (15). These investigators concluded that the Discovery NM 530c allows a more than 5-fold reduction in scan time and provides clinical perfusion and func- tion information equivalent to conventional dual-head SPECT MPI. In other reports, these same investigators made several suggestions regarding the Discovery NM 530c: optimal imaging times are $3 \mathrm{~min}$ and 2 min using conventional 1-d low-dose and high-dose protocols, respectively (16); breath triggering is facilitated, assisting in the discrimination of artifacts from true hypoperfusion similar to attenuation correction (17); left ventricular dyssynchrony can be assessed with a scan time of 5 min (18); attenuation correction can be performed using a CT transmission scan, yielding excellent clinical agreement when compared with conventional SPECT with attenuation correction (19); and image fusion with CT angiography can be performed (20). Figure 5 illustrates how CT attenuation correction can be used to correct for diaphragmatic attenuation.

\section{Detector Design Using Multiple Curved Nal Crystals (CardiArc)}

The next SPECT system to offer a totally new design was the CardiArc, manufactured by a company of the same name (21). This system uses 3 detectors, similar to conventional scintillation cameras, but the detectors are curved and side by side to cover a $180^{\circ}$ angle. Each detector consists of a curved $\mathrm{NaI}(\mathrm{Tl})$ crystal covered by an array of photomultiplier tubes. Horizontal collimation is defined using a series of thin lead sheets that are stacked vertically, with the gaps between sheets defining the hole apertures. Vertical collimation is achieved using a curved lead sheet with 6 vertical slits that fan back and forth during acquisition to obtain $180^{\circ}$ worth of data from multiple projections. The movement of these 6 slits is synchronized electronically with the 6 areas of the crystal that are imaging the photons passing through the slits. This design helps eliminate the

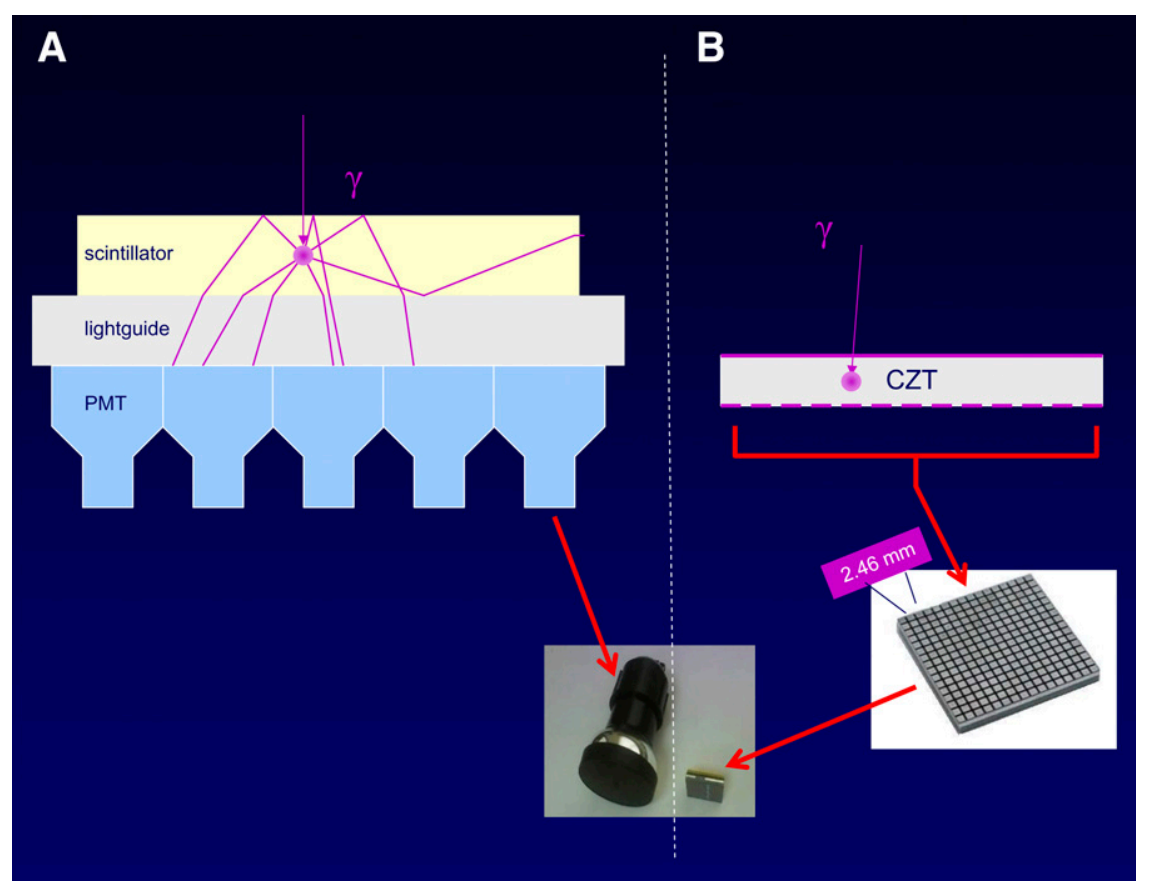

FIGURE 3. (A) Indirect radiation conversion with conventional SPECT detector. Nal (TI) crystal absorbs $\gamma$-rays from patient and converts its energy to visible photons, which are then converted to electrical pulses by entire array of photomultipliers. Sum of all pulses is energy information, and distribution of pulses is location of event in crystal. The large number of steps needed to reach these final data causes degradation of information as it is transferred from one mechanism to another, thus reducing both energy and spatial resolution of system. (B) Direct radiation conversion with $\mathrm{CZT}$ detector. Detector absorbs $\gamma$-rays from patient, directly converting its energy to charge carriers, which form electrical pulse with information on energy of event and with location being given by location of pixel within CZT detector where event took place. This more direct transfer of energy and location information results in better energy and spatial resolution than are obtained with conventional SPECT cameras. (Illustration modified from slides courtesy of Aharon Peretz.) 


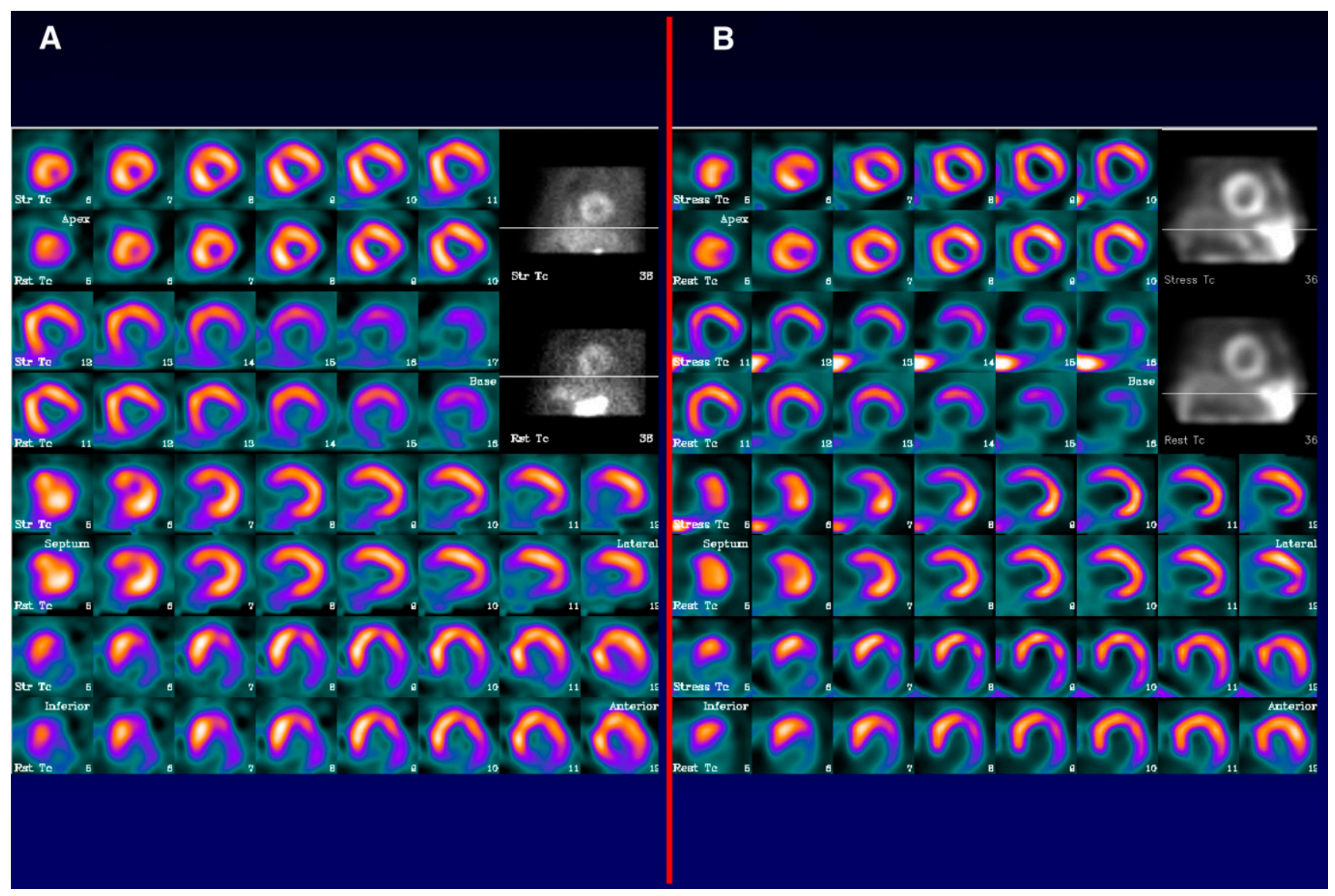

FIGURE 4. Abnormal imaging findings used to illustrate advantage of Discovery NM 530c SPECT system. Patient underwent rest-stress 99mTc-tetrofosmin MPI using $370 \mathrm{MBq}$ for rest and 1,110 MBq for stress. From top to bottom are shown short, vertical, and horizontal oblique-axis slices, with rest images appearing immediately below stress images. Black-and-white images are planar projections. (A) On conventional CardioMD SPECT system, rest and stress acquisitions were 14 and 12 min, respectively, and show fixed inferolateral hypoperfused wall. (B) On Discovery NM 530c SPECT system, rest and stress acquisitions were 4 and 2 min, respectively, and show high image contrast and partially reversible hypoperfused inferolateral wall.

overlap in acquisitions from adjacent slits. To date, no clinical validations of this device have been reported.

\section{DEVELOPMENTS IN ROTATING CAMERAS}

In addition to the radically new camera designs described above, other manufacturers and investigators have modified the electronics, system geometry, or collimation to significantly improve the imaging performance of rotating SPECT cameras.

\section{CsI Solid-State Multiple-Camera Design (Cardius 3)}

One of the first systems developed to take advantage of solid-state electronics and use more than 2 detectors simultaneously imaging the heart is the Cardius 3 XPO manufactured by Digirad. This commercial system uses 768 pixilated, thallium-activated $\mathrm{CsI}(\mathrm{Tl})$ crystals coupled to individual silicon photodiodes and digital Anger electronics to create the planar projection images used for reconstruction (7). Three detectors are fixed using a $67.5^{\circ}$ angular separation while patients are rotated through an arc of $202.5^{\circ}$ sitting on a chair with their arms resting above the detectors. Typical acquisition times for a study are $7.5 \mathrm{~min}$.
Manufacturers of this device claim up to $38 \%$ more count sensitivity than with conventional dual-head systems and comparable image quality. For attenuation correction, the system uses a monoenergetic fluorescent $\mathrm{x}$-ray source to create transmission photons, which are detected by the same 3 camera detectors positioned next to each other (22).

\section{Cardius-3 Validation}

In a large, multicenter trial using the nSPEED resolution recovery software and Digirad cameras, images of a subset of 189 patients were acquired using the Cardius 3 system and conventional doses and compared with conventional SPECT images. Using this combination, the study showed that a 5-min rest acquisition and 4-min stress acquisition yielded perfusion and function information from gated SPECT MPI studies that was diagnostically equivalent to full-time acquisition and 2-dimensional ordered-subset expectation maximization reconstructions (7).

\section{Multiple Confocal Collimator Design (IQ-SPECT)}

Siemens has reintroduced to the field the use of confocal collimators (now called IQ SPECT) (21). Previously used 


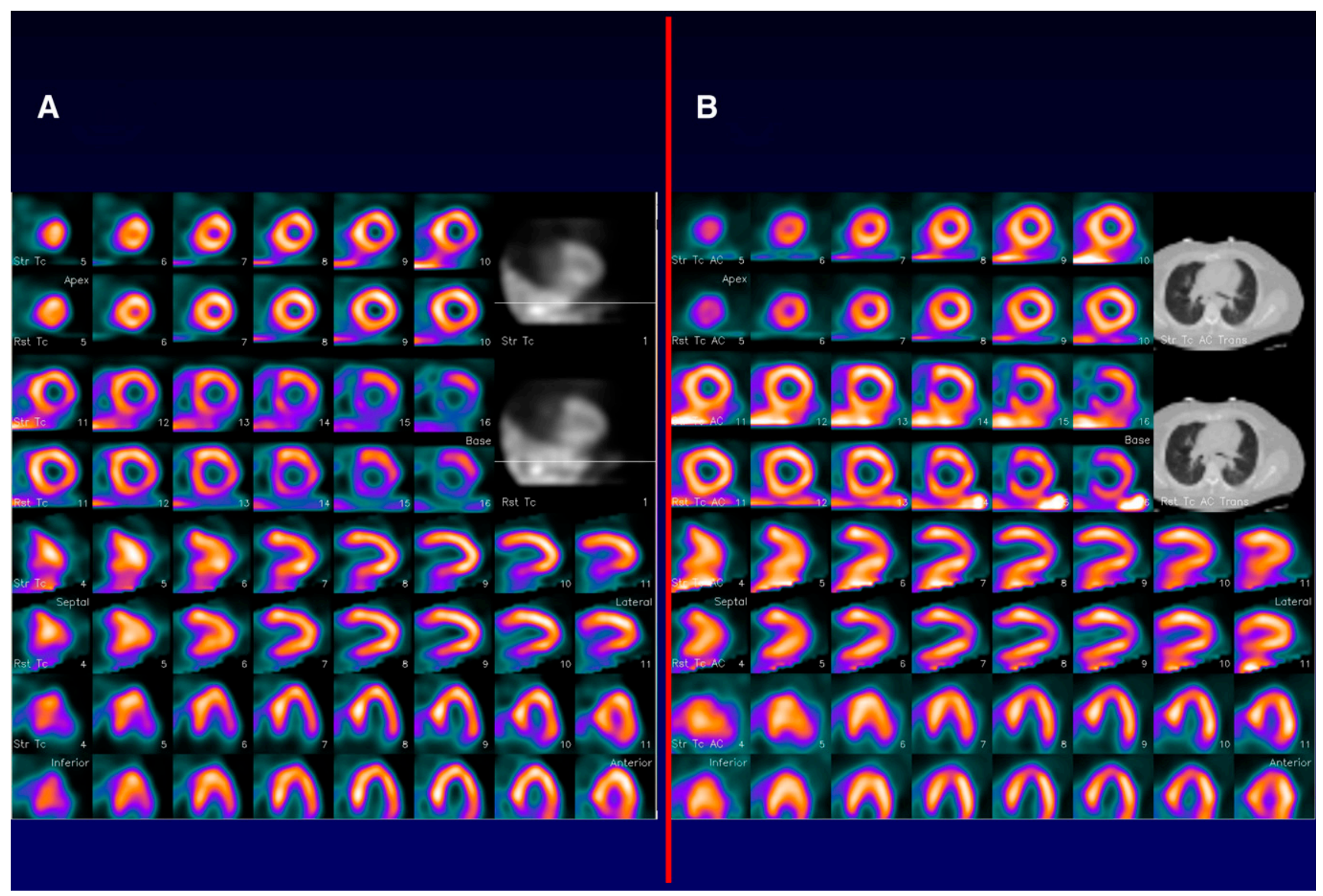

FIGURE 5. Normal imaging findings with diaphragmatic attenuation used to illustrate advantage of applying attenuation correction to Discovery NM 530c SPECT system. Patient underwent rest-stress $99 \mathrm{mT}$-tetrofosmin MPI using $370 \mathrm{MBq}$ for rest and 1,110 MBq for stress. From top to bottom are shown short, vertical, and horizontal oblique-axis slices, with rest images appearing immediately below stress images. (A) On Discovery NM 530c SPECT system, rest and stress acquisitions were 4 and 2 min, respectively, and show fixed inferobasal wall. Black-and-white images are planar projections. (B) With the addition of attenuation correction (using CT transmission shown in blackand-white images), increased tracer uniformity is seen throughout left ventricle, and inferior wall is seen to be normally perfused.

in single-head SPECT systems, 2 collimators are now mounted on their conventional dual-detector SPECT cameras, separated by $90^{\circ}$, and rotated around the patient to obtain a $180^{\circ}$ reconstruction arc. For increased sensitivity and resolution, the fields of view of these collimators are most convergent at their center, whereas the convergence is relaxed toward the edge of the field of view. The advantage of this approach is that it can be used by Siemens' existing dual-detector systems. Typical MPI acquisition times range from 4 to $5 \mathrm{~min}$.

\section{IQ-SPECT Validation}

A single-center clinical trial consisting of 54 patients concluded that 4-min-acquisition attenuation-corrected MPI with IQ-SPECT provided images of comparable quality and normalcy to 15-min-acquisition standard attenuationcorrected SPECT/CT (23).

\section{FAST-SPEED MPI: CLINICAL IMPLICATIONS}

The camera design and software improvements reviewed in this article reduce acquisition time for an MPI study anywhere from half the conventional time to a minimum 2-min acquisition. Clinically, these fast acquisitions would allow flexibility of acquisition protocols to reduce camera and patient total time, reduce cost, and increase patient comfort. Reduced acquisition times would also lead to decreased patient motion that results from translation, smearing, and breathing. Moreover, some of the count sensitivity increase may be traded for respiratory gating to eliminate the image smearing caused by chest motion and help reduce artifacts caused by the overlap of a hot liver and the left ventricular inferior wall. High count efficiency would also allow true stress acquisitions of myocardial function, as well as dynamic acquisition of SPECT tracers, similar to what is done with PET tracers. Dynamic imaging opens the door to the quantification of blood flow and coronary flow reserve, which can be used to detect disease earlier and to avoid interpretation of 3-vessel disease as normal (24). These technical breakthroughs in image reconstruction and truly heart-dedicated SPECT camera design have made high-speed MPI a reality (25).

\section{Reduced Dose Versus Increased Efficiency}

It is clear that the more efficient hardware and software of these imaging systems also allow for high-quality images 
obtained using a lower injected radiopharmaceutical dose and thus a decrease in the radiation dose absorbed by the patient and staff. This reduction in dose comes at an increase in acquisition time, even if the total time is less than what has traditionally been used in conventional systems. Because there currently are no financial incentives for using a lower radiopharmaceutical dose, a laboratory interested in just reducing costs would tend to opt for the most efficient protocol possible.

Recently, the American Society of Nuclear Cardiology published an information statement (26) recommending that laboratories use imaging protocols that achieve, on average, a radiation exposure of no more than $9 \mathrm{mSv}$ in $50 \%$ of the studies by 2014. Although there are many different protocols that may be implemented to achieve this goal, use of the more efficient hardware and software described here would greatly facilitate this achievement and allow for increases in efficiency over the imaging protocols used today.

\section{Protocol Flexibility Versus Standardized Imaging}

The widespread acceptance and success of MPI is owed in large part to the efforts of the American Society of Nuclear Cardiology (27) and the Society of Nuclear Medicine (28) to standardize the entire imaging procedure. These standards provide specific details for the many parameters included in MPI protocols and promote consistent high-quality images. Among these many parameters are the stress method, rest and stress dose injected, acquisition times, delay between injections, collimator type, reconstruction type, and filters. These guidelines $(27,28)$ have been developed mostly for rotating dual-head SPECT systems. As new ultrafast cardiocentric designs are commercialized, each with its own proprietary reconstruction implemented, it will become more challenging to continue to standardize protocols. Moreover, because these new designs allow for flexible imaging with a plethora of potential protocols (which may be patient-specific), our ability to implement standardization will be even more challenging. Thus, protocols may vary from a $1,110-\mathrm{MBq}$ stress-only MPI study with a CZT device with a 2-min acquisition, to the more conventional 14-min rest/12-min stress MPI studies with a 2- to 4-h delay between rest and stress injections, to simultaneous dual-isotope studies, to studies performed with $185 \mathrm{MBq}$ or less. The long-term success of nuclear MPI will depend on our ability to quickly converge on a discrete set of protocols that can be standardized for the entire field.

\section{Comparison with PET}

The clinical success of PET/CT oncologic imaging has promoted much wider availability of PET scanners, which may be used for cardiac imaging. This availability coupled with the perceived superiority of cardiac ${ }^{82} \mathrm{Rb}$ PET over cardiac SPECT due to superior spatial and contrast resolution, as well as more accurate attenuation correction, has resulted in a significant growth in cardiac PET. State-of-the- art PET scanners with new detectors and 3-dimensional imaging can provide high-quality ${ }^{82} \mathrm{Rb}$ cardiac images acquired using a lower patient radiation dose due to the higher count sensitivity and increased counting capabilities. High-quality transmission images from CT scans acquired using hybrid PET/CT systems provide accurate attenuationcorrected myocardial perfusion images except when there is misregistration between the sequentially acquired $\mathrm{CT}$ and PET studies $(29,30)$. The benefits of cardiac PET over conventional SPECT translate to improved detection and characterization of hypoperfused myocardium (31), although there is still a need for PET outcome data that match results from conventional SPECT (32). Moreover, a more pertinent comparison today is one between cardiac PET and the new ultrafast SPECT cameras, which are ushering in a new era in nuclear cardiology $(25,33)$.

\section{SUMMARY}

Dedicated cardiac SPECT cameras are undergoing a profound change in design for the first time in $50 \mathrm{y}$. The scintillation camera general-purpose design is being replaced with systems with multiple detectors focused on the heart yielding 5-10 times the sensitivity of conventional SPECT. Some of the designs also replace the NaI(Tl) crystal with solid-state electronic detectors with superior energy resolution. There are also significant innovations in reconstruction software incorporated into these newly designed systems that take into account the true physics of the SPECT reconstruction geometry to gain at least a factor of 2 in sensitivity. These innovations are resulting in shorter study times or reduced radiation doses to patients. Shorter study times promote easier scheduling, higher patient satisfaction, and, importantly, less patient motion during acquisition, which translates to higher-quality images. Some of these new systems are also ideally suited for dynamic applications facilitating measurements of coronary flow reserve. The fast acquisition also makes the hybrid SPECT/CT systems more practical since it allows the CT scanner to be used for a longer part of the day.

\section{ACKNOWLEDGMENTS}

Drs. Ernest Garcia and Fabio Esteves were investigators of a research grant funded by GE Healthcare to evaluate its Discovery NM 530c described in this article. Emory University also has received funding to evaluate the CardiArc device described in this article.

\section{REFERENCES}

1. Anger HO. Scintillation camera. Rev Sci Instrum. 1958;29:27-33.

2. Radon J. On the determination of functions from their integrals along certain manifolds [in German]. Berichte Saechsische Akademie der Wissenschaften. 1917;29:262-277.

3. Borges-Neto S, Pagnanelli RA, Shaw LK, et al. Clinical results of a novel wide beam reconstruction method for shortening scan time of Tc-99m cardiac SPECT perfusion studies. J Nucl Cardiol. 2007;14:555-565.

4. DePuey EG, Gadraju R, Clark J, Thompson L, Anstett F, Shwartz SC. Ordered subset expectation maximization and wide beam reconstruction "half-time" 
gated myocardial perfusion SPECT functional imaging: a comparison to "fulltime" filtered backprojection. J Nucl Cardiol. 2008;15:547-563.

5. Venero CV, Heller GV, Bateman TM, et al. A multicenter evaluation of a new post-processing method with depth-dependent collimator resolution applied to full and half-time acquisitions with and without AC. J Nucl Cardiol. 2009;16: 714-725.

6. Vija AH, Hawman EG, Engdahl JC. Analysis of a SPECT OSEM reconstruction method with 3D beam modeling and optional attenuation correction: phantom studies. Nucl Sci Symp Conf Rec. 2003;4:2662-2666.

7. Maddahi J, Mendez R, Mahmarian J, et al. Prospective multi-center evaluation of rapid gated SPECT myocardial perfusion upright imaging. J Nucl Cardiol. 2009;16:351-357.

8. DePuey EG, Bommireddipally S, Clark J, Thompson L, Srour Y. Wide beam reconstruction "quarter-time" gated myocardial perfusion SPECT functional imaging: a comparison to "full-time" ordered subset expectation maximization. J Nucl Cardiol. 2009;16:736-752.

9. Sharir T, Ben-Haim S, Merzon K, et al. High-speed myocardial perfusion imaging: initial clinical comparison with conventional dual detector anger camera imaging. J Am Coll Cardiol Imaging. 2008;1:156-163.

10. Sharir T, Slomka PJ, Hayes SW, et al. Multicenter trial of high-speed versus conventional single-photon emission computed tomography imaging: quantitative results of myocardial perfusion and left ventricular function. J Am Coll Cardiol. 2010;55:1965-1974.

11. Berman DS, Kang X, Tamarappoo B, et al. Stress thallium-201/rest technetium$99 \mathrm{~m}$ sequential dual isotope high-speed myocardial perfusion imaging. JACC Cardiovasc Imaging. 2009;2:273-282.

12. Ben-Haim S, Hutton BF, Van Grantberg D. Simultaneous dual-radionuclide myocardial perfusion imaging with a solid-state dedicated cardiac camera. Eur J Nucl Med Mol Imaging. 2010;37:1710-1721.

13. Garcia EV, Tsukerman L, Keidar Z. A new solid state ultra fast cardiac multidetector SPECT system [abstract]. J Nucl Cardiol. 2008;15(suppl):S3.

14. Esteves FP, Raggi P, Folks RD, et al. Novel solid-state-detector dedicated cardiac camera for fast myocardial perfusion imaging: multicenter comparison with standard dual detector cameras. J Nucl Cardiol. 2009;16:927-934.

15. Buechel RR, Herzog BA, Husmann L, et al. Ultrafast nuclear myocardial perfusion imaging on a new gamma camera with semiconductor detector technique: first clinical validation. Eur J Nucl Med Mol Imaging. 2010;37:773-778.

16. Herzog BA, Buechel RR, Katz R, et al. Nuclear myocardial perfusion imaging with a cadmium-zinc-telluride detector technique: optimized protocol for scan time reduction. $J$ Nucl Med. 2010;51:46-51.

17. Buechel RR, Pazhenkottil AP, Herzog BA, et al. Real-time breath-hold triggering of myocardial perfusion imaging with a novel cadmium-zinc-telluride detector gamma camera. Eur J Nucl Med Mol Imaging. 2010;37:1903-1908.
18. Pazhenkottil AP, Buechel RR, Herzog BA, et al. Ultrafast assessment of left ventricular dyssynchrony from nuclear myocardial perfusion imaging on a new highspeed gamma camera. Eur J Nucl Med Mol Imaging. 2010;37:2086-2092.

19. Herzog BA, Buechel RR, Husmann L, et al. Validation of CT attenuation correction for high-speed myocardial perfusion imaging using a novel cadmiumzinc-telluride detector technique. J Nucl Med. 2010;51:1539-1544.

20. Pazhenkottil AP, Husmann L, Kaufmann PA: Cardiac hybrid imaging with highspeed single-photon emission computed tomography/CT camera to detect ischaemia and coronary artery obstruction. Heart. 2010;96:2050.

21. Garcia EV, Faber TL. New trends in camera and software technology in nuclear cardiology. Cardiol Clin. 2009;27:227-236.

22. Bai C, Conwell R, Kindem J, et al. Phantom evaluation of a cardiac SPECT/VCT system that uses a common set of solid-state detectors for both emission and transmission scans. J Nucl Cardiol. 2010;17:459-469.

23. Corbett J, Meden J, Ficaro E. Clinical validation of attenuation corrected cardiac imaging with IQ-SPECT SPECT/CT [abstract]. J Nucl Med. 2010;51(suppl 2): $360 \mathrm{P}$.

24. Breault C, Roth N, Slomka PJ, et al. Quantification of myocardial perfusion reserve using dynamic SPECT imaging in humans [abstract]. J Nucl Cardiol. 2010;4(suppl):733.

25. Bonow RO. High-speed myocardial perfusion imaging: dawn of a new era in nuclear cardiology? J Am Coll Cardiol Imaging. 2008;1:164-166.

26. Cerqueira MD, Allman KC, Ficaro EP, et al. Recommendations for reducing radiation exposure in myocardial perfusion imaging. J Nucl Cardiol. 2010;17: 709-718.

27. Holly TA, Abbott BG, Al-Mallah M, et al. Single photon-emission computed tomography. J Nucl Cardiol. 2010;17:941-973.

28. Strauss HW, Miller DD, Wittry MD, et al. Procedure guideline for myocardial perfusion imaging 3.3. J Nucl Med Technol. 2008;36:155-161.

29. Gould KL, Pan T, Johnson NP, et al. Frequent diagnostic errors in cardiac PET/ CT due to misregistration of CT attenuation and emission PET images: a definitive analysis of causes, consequences, and corrections. J Nucl Med. 2007;48: 1112-1121.

30. Schuster DM, Halkar RK, Esteves FP, et al. Investigation of the emissiontransmission misalignment artifacts on rubidium-82 cardiac PET with adenosine pharmacologic stress. Mol Imaging Biol. 2008;10:201-208.

31. Bateman TM, Heller G, McGhie A, et al. Diagnostic accuracy of rest/stress ECG-gated Rb-82 myocardial perfusion PET: comparison with ECG-gated Tc-99m sestamibi SPECT. J Nucl Cardiol. 2006;13:24-33.

32. Di Carli MF, Hachamovich R. New technology for noninvasive evaluation of coronary artery disease. Circulation. 2007;115:1464-1480.

33. Di Carli MF, Hachamovitch R. Should PET replace SPECT for evaluating CAD? The end of the beginning. J Nucl Cardiol. 2006;13:2-7. 